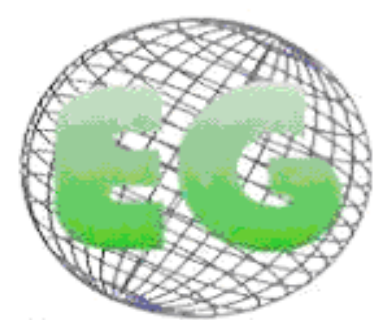

ISSN 1695-6141 $N^{\circ} 22$
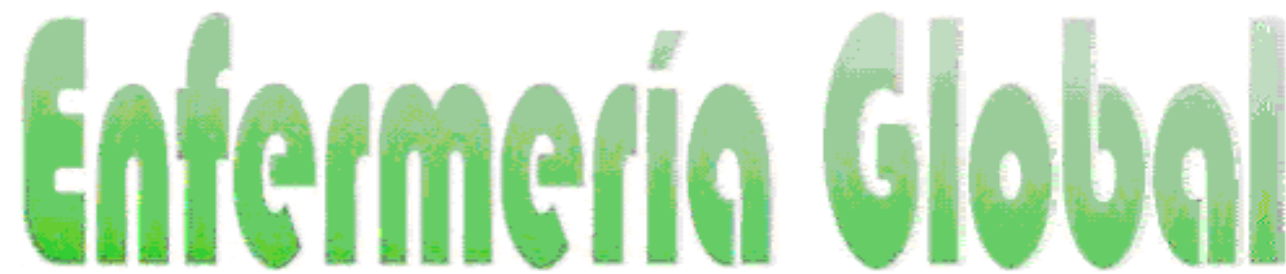

Revista electrónica trimestral de Enfermería

Abril 2011

www.um.es/egloball

\title{
PACIENTE ONCOLÓGICO TERMINAL: SOBRECARGA DEL CUIDADOR
}

\section{PACIENTE ONCOLÓGICO TERMINAL: SOBRECARGA DO CUIDADOR}

\author{
*Moreira de Souza, R., **Turrini, RNT. \\ *Graduando da Escola de Enfermagem da USP. " ${ }^{* *}$ Doutora em Enfermagem. Professora da Escola de \\ Enfermagem da Universidade de São Paulo. Brasil.
}

\author{
Palabras clave: Enfermo terminal; Oncología; Cuidadores; Enfermería \\ Palavras chave: Doente terminal; Oncologia; Cuidadores; Enfermagem. \\ Keywords: Medical Oncology; Terminally ill; Caregivers; Nursing.
}

\section{RESUMEN}

Se realizó un estudio de abordaje cualitativo para identificar los sentimientos vivenciados por el cuidador del paciente oncológico terminal. El artículo enfoca los sentimientos relativos a la sobrecarga.

Material y método: Entrevistados siete familiares cuidadores de pacientes oncológicos terminales atendidos en el ambulatorio de un hospital referencia en oncología con análisis de contenido de las entrevistas.

Resultados: Se identificaron tres categorías de sobrecarga: la física enfocó el agotamiento físico y el cuidar en el domicilio; en la mental se identificaron sentimientos de pérdida de autoestima, desesperanza, desánimo, tristeza y soledad; en la social, aspectos del papel femenino, alteración de la dinámica y renta familiar y acceso al servicio de salud.

Conclusiones: Las transformaciones en el cotidiano familiar y profesional del cuidador pueden llevarlo al agotamiento físico y emocional, con comprometimiento social. Los profesionales de salud deben prever soporte a los cuidadores en su domicilio, incluyendo educación dirigida al cuidado.

\section{RESUMO}

Realizou-se um estudo de abordagem qualitativa para identificar os sentimentos vivenciados pelo cuidador do paciente oncológico terminal. $O$ presente artigo enfocará os sentimentos relativos à sobrecarga. 
Material e método: Foram entrevistados sete familiares cuidadores de pacientes oncológicos terminais atendidos no ambulatório de um hospital referência em oncologia com posterior análise de conteúdo das entrevistas.

Resultados: Foi possível identificar três categorias de sobrecarga. A categoria sobrecarga física enfocou o esgotamento físico e o cuidar no domicílio; na categoria sobrecarga mental identificaramse sentimentos de perda da auto-estima, desespero, desanimo, tristeza e solidão; e na categoria sobrecarga social, aspectos do papel feminino, alteração da dinâmica familiar, renda familiar comprometida e acesso ao serviço de saúde.

Conclusões: As transformações no cotidiano familiar e profissional do cuidador podem levá-lo a exaustão física e emocional, com comprometimento social. Os profissionais de saúde devem prever suporte aos cuidadores em seu domicílio, incluindo educação voltada ao cuidado.

\section{ABSTRACT}

A quality study was developed to identify the emotions experienced by caregivers of terminally ill cancer patients. This study focused on the feelings related to their burnout.

Material and methods: Seven family caregivers of patients treated in the ambulatory care centre of an Oncology hospital were interviewed. Content analysis was used to identify reports of burnout among the caregivers.

Results. Three categories of burnout were observed. Physical overload was related to exhaustion and care at home. Mental overload was characterized by feelings of loss of self-esteem, despair, discouragement, sadness and loneliness. An exhaustion related to social aspects was also identified due to the female role, alteration in family functioning, compromised household income and access to the healthcare service.

Conclusions: Family and professional changes in daily lives of caregivers cause physical and mental exhaustion and social compromising. Healthcare workers should offer support to caregivers at home, including education for the one cared.

\section{INTRODUCCIÓN}

El cáncer es una enfermedad crónica-degenerativa, que viene creciendo entre la población mundial como consecuencia de una mayor expectativa de vida y de la exposición de los individuos a productos potencialmente cancerígenos.

Estadísticas en Brasil señalan al cáncer como la segunda mayor causa de muerte por enfermedad, dejando atrás únicamente a las muertes por enfermedades cardiovasculares, siendo más frecuente a partir de los treinta años de edad ${ }^{(1)}$. La mayoría de pacientes oncológicos diagnosticados se encuentra en la fase avanzada de la enfermedad y evolucionará hacia la muerte.

Así como el paciente oncológico terminal, la familia del paciente también pasa por varias transformaciones y por el enfrentamiento de la muerte. Esa interacción entre el paciente y su familia es una relación compleja, donde el enfermo sufre con las alteraciones de la familia y la familia sufre con las del enfermo.

Ante esas necesidades del paciente y de sus familiares, surgen los cuidados paliativos, definidos por la Organización Mundial de la Salud (OMS) como cuidados activos e integrales al paciente sin posibilidades de cura, con miras al control del dolor y de los demás síntomas 
y que tienen como objetivo la calidad de vida del paciente y de sus familiares ${ }^{(2)}$. El cuidado paliativo puede ser ejecutado en el nivel domiciliario, lo que algunas familias prefieren, debido a la mayor proximidad con el ente querido. Esa práctica puede contar con un equipo especializado, o concentrarse en un cuidador, que puede ser un pariente 0 amigo, denominado cuidador informal. Ese cuidador la mayoría de veces es escogido por deseo del paciente, por falta de opción entre los familiares o por la falta de un equipo especializado ${ }^{(3)}$.

El cuidador deja muchas cosas para estar con el enfermo, motivado por la búsqueda de la cura, pero afectado por desilusiones, sufrimientos y carga de trabajo brindada al paciente. Esas situaciones se intensificarán con el progreso de la enfermedad.

Con miedo de que algo malo suceda en su ausencia o por la misma preocupación con la falta de compañía del paciente, el cuidador tiende al aislamiento social, disminuyendo sus actividades y rutinas habituales, convirtiéndose en un prisionero de su hogar ${ }^{(4)}$.

Generalmente, el cuidador informal no se siente preparado y no tiene conocimiento suficiente para desempeñar esa función, lo que incrementa su agotamiento y lo lleva a dejar de lado sus propias necesidades de salud. Por otro lado, aquellos que se sienten más preparados para esa actividad sufren menos ${ }^{(5)}$.

Estudio $^{(6)}$ que evidenció el impacto sobre la salud física del cuidador, evaluó la relación entre el cuidar de un paciente terminal y el riesgo de muerte del cuidador por recrudecimiento del cuadro de enfermedades ya existentes. De todos los casos investigados, se observó mayor riesgo sobre cuidadores con esposos(as) dependientes y que se sentían sobrecargados con las tareas administrativas. Esa sobrecarga de diferentes factores aumentó el riesgo de morbilidad y de mortalidad en el cuidador.

La definición de sobrecarga incluye dos aspectos fundamentales: el objetivo y el subjetivo. La sobrecarga objetiva corresponde a los resultados negativos concretos y visibles del papel del cuidador y la subjetiva se refiere a las sensaciones que el cuidar desencadena, a la evaluación personal de los familiares sobre la situación ${ }^{(7)}$.

Algunas investigaciones muestran que cuidadores con alto grado de sobrecarga son más fácilmente afectados por la depresión, con una frecuencia descrita en media de $30 \%$ de los cuidadores observados ${ }^{(8)}$.

Se realizó una investigación para identificar los sentimientos vividos por el cuidador del paciente oncológico terminal. En el análisis de los datos fueron identificados dos temas: el proceso de morir y la sobrecarga del cuidador. El presente trabajo tiene por objetivo presentar los resultados relacionados a la sobrecarga.

\section{MATERIAL Y MÉTODOS}

Estudio exploratorio, de abordaje cualitativo, que utilizó una muestra de conveniencia constituida por siete cuidadores de pacientes oncológicos terminales atendidos en el servicio ambulatorio de un hospital público docente de atención terciaria a la salud, los cuales aceptaron participar del estudio. El criterio de inclusión fue ser cuidador principal del paciente oncológico con cáncer avanzado, y este fue definido como aquel con Karnofsky Performance Status (KPS) abajo de 50. El KPS evalúa el grado de deterioro del estado funcional del paciente. 
De los entrevistados, seis eran de sexo femenino, y uno masculino. En relación al grado de parentesco: cuatro eran hijos, dos eran esposas y uno era padre.

Procedimiento de recolección: los datos fueron recolectados por medio de la entrevista no-dirigida por utilizar algunos dispositivos analíticos como la atención fluctuante y la regla de libre asociación, que consiste en exprimir indiscriminadamente todos los pensamientos que amparan al espíritu, ya sea a partir de un elemento determinado, o de forma espontánea ${ }^{(9)}$.

La pregunta norteadora de la entrevista fue: ¿ "Cuénteme cómo es para usted cuidar de su familiar?". Las entrevistas fueron grabadas con la autorización del entrevistado y a partir de discurso inicial del entrevistado, el investigador estimuló el tema en cuestión.

Las entrevistas fueron realizadas en un local privado, con la finalidad de garantizar el sigilo y privacidad. La duración de las entrevistas osciló entre diez y quince minutos. El proyecto de investigación fue aprobado por la Comisión de Ética en Investigación de la Escuela de Enfermería de la Universidad de Sao Paulo y fue solicitada autorización de la Institución de Salud para la realización de la investigación y utilización de la grabadora para las entrevistas.

Análisis de los datos: A partir de la trascripción fiel de las cintas grabadas durante la entrevista, se realizó el análisis de contenido para identificar los sentimientos vivenciados por los cuidadores de pacientes oncológicos terminales. Después de repetidas lecturas del material, fue posible identificar dos temas proceso de morir y sobrecarga del cuidador. En este estudio serán presentados los resultados relacionados a la sobrecarga del cuidador.

Los entrevistados fueron identificados en el análisis de contenido por un código que utiliza la letra "S" de sujeto y el número de la respectiva entrevista.

\section{RESULTADOS Y DISCUSIÓN}

En la lectura de las narraciones fue posible identificar sentimientos relacionados con tres categorías de sobrecarga: física, mental y social. Teniendo en cuenta los diferentes aspectos involucrados en cada una de ellas se establecieron subcategorías de análisis.

\section{Sobrecarga física}

En esta categoría se destacaron el agotamiento físico, proveniente de las acciones de cuidar, y las dificultades de cuidar en el domicilio.

El esfuerzo continuado requerido en el proceso de cuidar, aunado a las características demográficas y a las responsabilidades sociales y familiares, son factores que conducen al deterioro físico del cuidador ${ }^{(10)}$.

Agotamiento físico. A medida que el paciente se vuelve dependiente por el progreso de la enfermedad y debilidad física, el cuidador pasa a asumir actividades relacionadas a la atención de sus necesidades fisiológicas como nutrición, higiene y comodidad. Al principio, esas actividades son razonablemente toleradas, pero luego de un período de tiempo ellas sobrecargan los quehaceres diarios del cuidador y provocan el desgaste físico.

En las siguientes entrevistas es posible notar la cantidad de tareas que desarrolla el cuidador para cuidar del paciente en la atención de sus necesidades fisiológicas básicas, lo que también denota el grado de dependencia del paciente. 
Porque ahí, ahí hay los cambios de pañales continuos, tenemos que cambiar continuamente los pañales... (S2)

Lo bañamos, lo limpiamos, yo y mi mamá y mi hermana, ¿no? Nosotros tres, entonces, nosotros hacemos todo para él. Rasurar la barba... todo lo que él necesita [...] es comida, todo en la mano porque él está en cama, ¿no?... (S3)

No siempre la situación familiar permite que más personas dividan las tareas de cuidador. Datos de la literatura evidencian que el cuidador acaba desarrollando múltiples funciones y, muchas veces, se convierte en el único cuidador ${ }^{(11)}$.

En la dedicación integral al paciente, el cuidador tiende a dejar de lado sus propias necesidades fisiológicas y de salud, exponiéndose a estilos de vida poco saludables, como se muestra:

No duermo de noche porque lo cuido [...] a cualquier hora que él se mueve en la cama yo estoy despierta[...] tengo problemas de laberintitis... tengo sesenta y tres años... no estoy durmiendo, ... no estoy alimentándome (S7).

La literatura muestra que el cuidador informal no tiene horarios ni períodos de descanso regulares, promoviendo un agotamiento físico intenso y solamente comunica sus propios problemas de salud cuando se ven agravados ${ }^{(12)}$.

Es común en las situaciones de enfermedad en la familia que el cuidador retrase sus necesidades de salud por creer que son menos importantes o porque no consigue reservar una parte de su tiempo para cuidar de sí mismo y buscar atención médica. Es más, el cuidador puede no tener con quien dividir sus tareas para que pueda buscar atención médica para resolver su problema de salud. En la mayoría de casos, los cuidadores familiares no tienen recursos financieros para contratar servicios especializados 0 cuidadores para aliviar la sobrecarga.

La práctica profesional muestra un elevado número de cuidadores de la tercera edad que no tienen la vitalidad para cuidar de personas dependientes, aunque desde el punto de vista emocional, probablemente, estén más preparados que las personas más jóvenes.

El adulto mayor es aquel que puede dedicarse al cuidado por estar fuera del mercado laboral, pese a su condición física o financiera cuestionables para hacerlo. Además de eso, existen casos de personas que no tienen hijos o fueron abandonados por ellos. A menudo, en casos de enfermedades terminales, los familiares se alejan para no hacer frente a la enfermedad y la muerte y las acciones de cuidar recaen sobre el cónyuge, a pesar que en este estudio el principal parentesco del cuidador era ser hija (o).

Cuidado en el Domicilio. La dificultad para el cuidado en el domicilio podría atribuirse a factores como la complejidad del propio cuidado, la ausencia de un equipo de apoyo para la atención en el domicilio, al grado de escolaridad del cuidador y a los aspectos culturales sobre el cuidado administrado y el morir en casa.

Es difícil para el cuidador proveer el cuidado en el domicilio, por no contar con la ayuda o por falta de competencia técnica cuando el paciente utiliza dispositivos médicos para el mantenimiento de algunas de sus necesidades fisiológicas básicas. 
Muchas veces, el paciente en estado avanzado de la enfermedad, necesita de apoyo nutricional enteral y el cuidador precisa aprender a prepararlas, administrarlas y mantener la permeabilidad del sistema de infusión de la dieta.

Cuanto mayor sea la gravedad del paciente, mayor es la necesidad de cuidado especializado, como se ve en la siguiente frase:

...tenemos que lavar el tubo de ella, sacarlo para desobstruirlo constantemente, tenemos que darle nebulización, tenemos que cambiar la curación... (S2)

El cuidado especializado necesita ser aprendido, pues esta habilidad no se adquiere en el ambiente familiar. La literatura muestra que la falta de conocimiento por parte del cuidador aumenta la sobrecarga, ya que el saber hacer exige preparación teórica y práctica ${ }^{(13)}$.

Las frases siguientes indican la necesidad que el cuidador tiene de adquirir conocimiento sobre mejores formas de cuidar, pero conciliar todos sus papeles sociales con las tareas de cuidador no favorece el tiempo para el aprendizaje:

A medida que van apareciendo las necesidades, nosotros vamos aprendiendo... ah... ¿no?... entonces nosotros vamos aprendiendo de nuevo... porque es una situación nueva al comienzo (S3)

[...] a pesar de que yo coja un libro, y comience a buscar, yo voy...pero últimamente estoy sin tiempo, porque yo tengo un bebé, ¿no? (S5)

Por más que el cuidador se empeñe en el cuidado y haga lo mejor por el otro, él siente que su esfuerzo es en vano delante de la evolución de la enfermedad y de la finitud de la vida. Muchas veces cree fielmente que no hay ningún resultado de su trabajo, como se evidencia en la siguiente oración:

Tenemos que hacer la comidita, tenemos que darle la comida seis veces al día, pero no esta sirviendo de nada, ella está disminuyendo cada día que pasa... (S2)

Un estudio muestra la necesidad de mayor acompañamiento de los cuidadores por el equipo de salud, ya que el cuidado exige una preparación emocional y técnica ${ }^{(13)}$.

Una de los temas principales y más estresantes en el cuidado domiciliario es el manejo del dolor en el paciente terminal, como ya fue informado en otro estudio ${ }^{(14)}$. Se pueden ver en los discursos la dificultad del cuidador para aliviar el dolor del enfermo, incluso con la administración de los medicamentos.

Nunca es suficiente, por más que tú intentes aliviar el dolor que ella está sintiendo, no es suficiente... creo que es difícil... (S2)

Es difícil para mi, porque la veo con dolor, la veo llorando, ahí tu llevas su medicación, y luego vuelve el dolor... no sé por qué... no pasa... ¿entendió?... (S5)

Algunos cuidadores, además de considerar el cuidado agotador en el domicilio, también expresan que el cuidado brindado por profesionales habilitados garantizaría una mejor atención al paciente, como en las declaraciones que siguen: 
No es que en casa nadie tenga instrucción [...] para cuidar de mi hijo [...] porque él tiene problemas de cabeza y es muy difícil cuidar de él en casa [...] lo ideal sería un enfermero, un local apropiado para que el pueda estar cerca de la casa (S6)

El paciente con cáncer puede tener otras patologías lo que convierte la acción de cuidar en más agotadora, principalmente en los casos de compromiso del estado mental.

Testimonios recogidos por expertos en pacientes terminales ${ }^{(15)}$ muestran que el paciente terminal se siente mejor en su domicilio y deben gozar del contacto familiar en la fase terminal de la vida. Sin embargo, no siempre los familiares comparten esta percepción.

La mayoría de las veces lo que determina el que no se ingrese al paciente es la ausencia de camas hospitalarias y el costo de ese paciente para los servicios de salud. Es importante que el equipo de salud esté sensibilizado para percibir si la familia acepta atender al paciente en casa o si tiene condiciones emocionales, físicas, financieras, e inclusive de espacio físico para asumir el cuidado de un paciente terminal en el domicilio.

\section{Sobrecarga mental}

Cuidar de un paciente terminal puede traer sobrecarga emocional, estrés, miedo, agotamiento, ansiedad y menor satisfacción con la vida.

En el análisis de los discursos es evidente que muchos aspectos de la sobrecarga mental son resultado del desgaste físico. El hecho de sentirse frustrado en la obtención de resultados positivos del cuidar referentes al mantenimiento de la vida y del bienestar del paciente terminal moviliza sentimientos negativos de auto-desvalorización y de impotencia.

Pérdida de la autoestima. La falta de reconocimiento de las acciones desarrolladas en el acto de cuidar compromete la autoestima del cuidador como evidencia el discurso siguiente:

Hay días en que siento que no sirvo para nada, todo lo que hago está mal, luego viene una persona cualquiera de la calle, quien sea, viene y le dice a ella haz esto $o$ haz aquello, ahí ella lo hace [...] pues conmigo [...] está todo equivocado (S1)

La literatura muestra que el trabajo del cuidador es comúnmente poco reconocido, de bajo estatus y hasta invisible ${ }^{(12)}$.

La persona se siente desvalorizada, cree no ser capaz de desarrollar el papel de cuidador y puede no ser capaz de comprender las reacciones del paciente terminal, principalmente ante el desgaste que siente con la situación.

El paciente terminal vive períodos de rabia por la pérdida de su papel en el núcleo familiar, social o profesional, al no tener más control sobre la satisfacción de sus necesidades ${ }^{(16)}$. Esta reacción termina comprometiendo la relación con el cuidador que desarrolla sentimientos de auto-desvalorización por no ser reconocido su trabajo y dedicación.

Desesperación. El nivel de tensión emocional del cuidador es mayor del que existe en la población en general. La desesperación es señalada como un síntoma que acompaña a la depresión y que se presenta en situaciones de sobrecarga psíquica con aumento de la ansiedad del cuidador ${ }^{(8)}$. 
Acompañar al enfermo en su sufrimiento genera en el cuidador un sentimiento de desesperación y una dificultad en continuar acompañando al paciente en ese proceso de morir, lo que se muestra evidentemente en estas oraciones:

Nos desespera también... entonces... es muy, pero muy difícil [...] mantenerse, estar ahí... (S2)

Es muy difícil [...] (voz muy temblorosa y llorosa) hay horas en que siento un dolor tan grande... siento aquella angustia [...] Me da desesperación verlo así de esa forma... sintiendo mucho dolor [...] esta siendo muy difícil para mi [...] estoy peor que él (S7)

Vivenciar el sufrimiento del paciente puede ser una tarea difícil y requiere una preparación del cuidador para ese enfrentamiento, no siempre presente. El cuidador en la fase terminal del enfermo necesita convivir con la situación del dolor de difícil control y la decadencia de la persona sin que pueda interrumpir ese proceso, además de la constante amenaza de muerte en cualquier momento.

Es muy pero muy difícil, muy doloroso. Porque uno ve poco a poco, que ellos se van, ¿no? Y no hay nada que hacer... cómo hacer para que vuelva (voz temblorosa y llorosa) [...] Sólo Dios... puede... y (respira profundo y seca las lágrimas)... es mucho sufrimiento (pausa) mucho sufrimiento (S2)

En el discurso de S2, se percibe un intento de comprender la razón de ese proceso por la creencia religiosa. A pesar de buscar consuelo en la religión, los dolores de la pérdida y de la muerte cercana están presentes en todo momento, lo que intensifica el sufrimiento del cuidador. Aunque el sufrimiento persista, existen estudios que demuestran un menor desarrollo de depresión y sufrimiento psicológico en cuidadores religiosos ${ }^{(17)}$.

Forma parte del desarrollo espiritual de cada individuo comprender el dolor del otro, sentir compasión y conseguir pasar por esas experiencias sin sentir el dolor por el otro. Esto es importante, ya que sólo así el cuidador tendrá las fuerzas para llevar a cabo esa misión tan difícil de ayudar al otro a morir y permitir que el paciente se sienta reconfortado para hablar sobre la muerte.

Desaliento y Tristeza. Datos de la literatura también indican al desaliento y a la tristeza como manifestaciones resultantes de la depresión ${ }^{(8)}$.

El desaliento y la tristeza impregnan la vida cotidiana del cuidador:

Luego pasa y viene un nuevo día, a veces estamos desanimados (S3)

Yo ando muy triste... (S7)

Algunos estudios demuestran que la sobrecarga proveniente de los cuidados, asociada a factores como la visión negativa del cuidador sobre la enfermedad, la ausencia de una red de soporte social y la difícil relación con el paciente, aumentan considerablemente los sentimientos de desaliento y tristeza. Esos factores desenvuelven en el cuidador una menor satisfacción con la vida ${ }^{(18)}$.

La expresión de tristeza puede tener dos fuentes: una en el enfrentamiento de la finitud de la vida y la otra en la pérdida de "su propia vida". En la mayoría de los casos, las acciones de cuidar ocupan todo el tiempo de la persona, que ya no tiene un momento más para cuidar de 
sí mismo y escuchar sus propias necesidades sean estas físicas, afectivas, familiares o sociales.

Soledad. Inmersa en las tareas del cuidado, generalmente en tiempo integral, el cuidador puede aislarse de su relación con otras personas, no teniendo con quien conversar y dividir su sufrimiento.

Yo no tengo con quien desahogarme (llanto intenso)... (S7)

Datos de la literatura demuestran que el cuidador se aísla socialmente, y por esa actitud sufre de soledad en su vida cotidiana ${ }^{(4)}$.

El cuidador puede sentirse en la obligación de contener sus emociones, por creer que el paciente se sentirá mucho peor si percibe su sufrimiento y su sobrecarga, como se percibe en el siguiente discurso:

Ahí también uno no puede estar llorando cerca de la persona, porque ahí ella, ¿no? La persona ya no está bien, y si tú no te calmas... a veces tú estas viendo que ella está mal, no se puede aguantar... si tú desahogas junto con la persona... yo creo que empeoran las cosas, ¿no? (S5)

Sin mostrar sus emociones y lejos de la vida social, el sentimiento de soledad es cada vez más presente, y puede manifestarse bajo la forma de ira e impaciencia en el cuidado.

\section{Sobrecarga Social}

Papel femenino. Con relación al cuidado, la mujer puede sentirse presionada a asumir el papel de cuidadora, y puede ser vista como responsable de esos cuidados, debido a las construcciones sociales de género. La frase siguiente ilustra esa visión:

Pero es difícil cuidar, ¿vio?... la exigencia es muy grande, muy grande... no tanto de ella... sino de la familia... porque sólo yo soy mujer los demás son todos varones, y todos están casados, entonces sólo quedo yo... (S1)

La literatura también refiere que algunas mujeres adoptan para sí la actividad de cuidadora por considerar que es una tarea femenina ${ }^{(19)}$.

Socialmente, cabe a la mujer soltera asumir los cuidados de los familiares en casos de enfermedad, por asumir que tiene mayor disponibilidad. Por no haber otro cuidador 0 alternativa para el cuidado del paciente terminal, la persona permanece en esa función por obligación y con sufrimiento por actuar contra su voluntad.

[...] se hace porque tiene que hacerse, porque uno, uno tiene que hacerlo... porque no hay otra alternativa... (voz temblorosa y ojos llorosos) (S2)

Se establece así un conflicto interno entre la necesidad de cuidar por cuestiones sociales / éticas / familiares y el disgusto por el cuidado o el enfrentamiento de la muerte.

Alteración de la dinámica familiar. Independientemente de la gravedad, la presencia de un enfermo en casa siempre altera la dinámica familiar. Los efectos se sienten más cuando la condición socio-económica del cuidador es menor y el enfermo tiene mayor dependencia. En 
el mejor de los casos, la familia es capaz de unirse en ese momento, establecer rutinas de rotación de las actividades y servir de soporte al cuidador.

Según la literatura, mientras la enfermedad avanza, las relaciones familiares se alteran y se complican las rutinas cotidianas ${ }^{(12)}$.

Para estar con el paciente, el cuidador deja su propia familia y se pone de acuerdo con otros familiares para desempeñar su función, como se menciona a continuación:

Uno cuida un día, ahí la otra va y cuida al otro día, cuando uno necesita salir, la otra se queda... ahora yo vine para acá y dejé mis dos hijas con mi hermana, la otra está trabajando (S2)

Yo me dediqué mucho durante el año pasado [...] casi abandoné mi hogar (S4)

Con frecuencia, el abandono del hogar y de la vida privada desestructuran la familia del cuidador. Con la muerte del paciente, el cuidador se depara con la pérdida y con la necesidad de recuperar su hogar y los lazos familiares que se debilitaron.

Ingreso familiar comprometido. Pueden existir conflictos del cuidador con su trabajo, que muchas veces se ve obligado a faltar al servicio o hasta abandonar el empleo ${ }^{(20)}$.

Por más que el cuidador logre organizar su dinámica familiar, no siempre existe la misma comprensión en el ambiente de trabajo formal del cuidador, principalmente cuando el cuidador necesita ausentarse del servicio para acompañar al paciente a las consultas y tratamientos ambulatorios, como se evidencia en la siguiente oración:

Porque... el empleo no quiere saber... ¿no? Si tú andas faltando... porque son dos días... entonces, quiere decir que tú perdiste... porque uno tiene que venir para acá, y aquí es un poco lento, ¿ verdad? (S5)

Otras veces, el ausentismo por motivos de enfermedad en la familia no es aceptado por el empleador y el cuidador pierde su empleo comprometiendo más aún la economía familiar:

Entonces, ya perdí hasta mi empleo por causa de eso... (traer el paciente a la consulta) entonces, es muy complejo... (S6)

La dificultad financiera es un agravante del estrés del cuidador y de la familia. Los cuidadores con bajos ingresos y de pacientes con alto grado de dependencia son los más afectados. Esta situación afecta en la adquisición de medicamentos y en la compra de alimentos para el paciente y su familia, como señalan los relatos:

Está muy difícil, difícil de verdad (voz temblorosa y llorosa) Yo hasta... doy gracias a Dios cuando él está internado, porque aquí él tiene alimentación [...] iAy!.. cómo es difícil... problema de... de comprar medicamentos para él... es dar el alimento... que yo no tengo... no tengo cómo... lo más difícil para mí es eso... (S7)

La situación socio-económica del paciente y su familia se opone al intento de estimular a la familia para mantener al enfermo terminal en el domicilio con la justificación de que tendría una mejor calidad de vida y se sentiría acogido por los familiares en ese momento de despedida. Lo que se percibe en las narrativas de los cuidadores es que ese momento 
puede ser de mucha angustia por la dificultad de garantizar la subsistencia del enfermo y de la propia familia.

Acceso al servicio de salud. Las dificultades de acceso al servicio de salud, como distancia y medio de transporte, pueden contribuir a un mayor desgaste del cuidador, hecho que se evidencia en los siguientes relatos:

Me sacaba completamente de nuestra rutina diaria, porque yo lo acompañaba, lo levantaba diariamente a las cuatro y media de la mañana (S4)

Yo tuve que pedirle al vecino que lo traiga [...] Está difícil porque ella no aguanta, mientras estaba aguantando nosotros veníamos de ómnibus [...] Entonces quiere decir que yo estoy dependiendo... de estar corriendo detrás de alguien que pueda estar trayéndola aquí (S5)

La mayor parte de la población atendida en el hospital donde se realizó este estudio tiene bajo poder adquisitivo, vive lejos y no es raro que dependan de más de una movilidad para llegar al servicio de salud.

Se indican como limitaciones de este estudio, el hecho de que el cuidador en el momento de la entrevista no estaba totalmente tranquilo, ya que las entrevistas no fueron marcadas con anticipación y la mayoría de ellos estaban preocupados con la llamada para la consulta. Los datos obtenidos en este trabajo representan la población atendida en el hospital de estudio, no siendo generalizados para toda la población, dado que algunos hallazgos, principalmente los relacionados a la sobrecarga social, pueden variar de acuerdo con el nivel socioeconómico de los entrevistados.

\section{CONCLUSIONES}

El estudio permitió identificar los diferentes sentimientos del cuidador del paciente oncológico terminal, referentes a la sobrecarga y quedó probado que las transformaciones en el cotidiano familiar y profesional del cuidador pueden llevar a la persona al agotamiento físico y emocional, con repercusiones para su estado de salud.

Factores como la falta de preparación para el cuidado y la ausencia de soporte familiar y profesional sobrecargan al cuidador. Una especial atención del equipo multiprofesional en la evaluación de las condiciones de la familia para el cuidado del paciente terminal en el domicilio y un entrenamiento de los cuidadores para que puedan asumir determinados cuidados de baja complejidad que requieren conocimiento técnico de enfermería, son intervenciones necesarias para la asistencia domiciliaria del paciente terminal.

El apoyo del equipo de salud permitirá que el cuidador se sienta acogido para expresar sus sentimientos y angustias y posibilitará que continúe ejerciendo su papel con el menor sufrimiento posible hasta la muerte del paciente.

\section{REFERENCIAS}

1. Ministério da Saúde (BR). Taxa de mortalidade por neoplasia maligna no Brasil. Data SUS; (citado 2007 abr 26). Disponível em: http://www.datasus.gov.br.

2. World Health Organization. Cancer pain relief and palliative care report. Geneva;1990. 
3. Wennman-Larsen A, Tieshelman C. Advanced home care for cancer patients at the end of life: a qualitative study of hopes and expetations of family caregivers. Scand $J$ Caring Sci 2002;16:240-247.

4. Proot IM, Abu-Saad HH, Crebolder HFJM, Goldsteen M, Luker KA, Widdershoven GAM. Vulnerability of family caregivers in terminal palliative care at home; balancing between burden and capacity. Scand J Caring Sci 2003;17:113-121.

5. Given B, Sherwood PR. Family care for older person with cancer. Semin Oncol Nurs 2006;22(1):43-50.

6. Schultz R, Beach SR. Caregiver as a risk factor for mortality: The caregiver health effects study. JAMA 1999;282: 2215-9.

7. St.Onge $\mathrm{M}$, \& Lavoie $\mathrm{F}$. The experience of caregiving among mothers of adults suffering from psychotic disorders: factor associated to their psychological distress. Am J Community Psychol 1997;25(1):73-94.

8. Rezende VL, Derchain SFM, Botega NJ, Sarian LO, Vial DL, Morais SS. Depressão e ansiedade nos cuidadores de mulheres em fase terminal de câncer de mama e ginecológico. Rev Bras Ginecol Obst 2005;27(12): 737-43.

9. Thiollent M. Crítica metodológica, investigação social e enquête operatória. Polis, 1982.

10. Andrade OG, Rodrigues RAP. O cuidado familiar ao idoso com seqüela de acidente vascular cerebral. Rev Gaúch Enferm 1999; 20(2): 90-109.

11. Floriani CA, Schramm FR. Cuidador do idoso com câncer avançado: um ator vulnerado. Cad Saúde Pública 2006;22(3):557-534.

12. Benjumea C C. Cuidado familiar em condiciones crônicas: uma aproximación a la literatura. Texto Contexto Enferm 2004;13(1):137-49.

13. Machado ALG, Freitas CHA, Jorge MSB. O fazer do cuidador familiar: significados e crenças. Rev Bras Enferm 2007; 60(5):530-4.

14. Vallerand $\mathrm{AH}$, Collins-Bohler D, Templin T, Hasenau SM. Knowledge of and barriers to pain management in caregivers of cancer patients receiving homecare. Cancer Nurs 2007;30(1):31-37.

15. Klubber-Ross E. Viver até dizer adeus. São Paulo: Pensamento; 2005

16. Klubber-Ross E. Sobre a morte e o morrer. 8ª São Paulo: Martins-Fontes; 1998.

17. Fenix JB, Cherlin EJ, Prigerson HG, Johnson-Hurzeler R, Kasl SV, Bradley EH. Religiousness major depression among bereaved family caregivers: a 13-month follow-up study. J Palliat Care 2006; 22 (4) :286-292.

18. Pitceathly C, Maguire $P$. The psychological impact of cancer on patients' partners and other key relatives: a review. Eur J Cancer 2003; 39: 1517-24. 
19. Silva L, Galera SAF, Moreno V. Encontrando-se em casa: uma proposta de atendimento domiciliar para famílias de idosos dependentes. Acta Paul Enferm 2007;20(4):397-403.

20. Grunfeld E, Coyle D, Whelan T, Clinch J, Reyno L, Earle CC, Willan A, Viola R, Coristine M, Janz T, Glossop R. Family caregiver burden: results of a longitudinal study of breast cancer patients and their principal caregivers. Can Med Assoc J 2004;170(12):1795-801. 